\title{
THEMIS satellite observations of hot flow anomalies at Earth's bow shock
}

\author{
Christina Chu ${ }^{1}$, Hui Zhang ${ }^{1}$, David Sibeck ${ }^{2}$, Antonius Otto ${ }^{1}$, QiuGang Zong ${ }^{3}$, Nick Omidi ${ }^{4}$, James P. McFadden ${ }^{5}$, \\ Dennis Fruehauff ${ }^{6}$, and Vassilis Angelopoulos ${ }^{7}$ \\ ${ }^{1}$ Geophysical Institute, University of Alaska Fairbanks, 903 Koyukuk Dr, Fairbanks, AK 99775, USA \\ ${ }^{2}$ NASA Goddard Spaceflight Center, Greenbelt, Maryland, USA \\ ${ }^{3}$ Institute of Space Physics and Applied Technology, Peking University, Beijing, China \\ ${ }^{4}$ Solana Scientifica, San Diego, California, USA \\ ${ }^{5}$ Space Sciences Laboratory, University of California Berkeley, Berkeley, California, USA \\ ${ }^{6}$ Institute of Geophysics and Extraterrestrial Physics, Technische Universität Braunschweig, \\ Braunschweig, Germany \\ ${ }^{7}$ Department of Earth, Planetary, and Space Sciences, University of California Los Angeles, \\ Los Angeles, California, USA
}

Correspondence to: Hui Zhang (hzhang14@alaska.edu)

Received: 27 December 2016 - Revised: 2 February 2017 - Accepted: 3 February 2017 - Published: 17 March 2017

\begin{abstract}
Hot flow anomalies (HFAs) at Earth's bow shock were identified in Time History of Events and Macroscale Interactions During Substorms (THEMIS) satellite data from 2007 to 2009. The events were classified as young or mature and also as regular or spontaneous hot flow anomalies (SHFAs). The dataset has 17 young SHFAs, 49 mature SHFAs, 15 young HFAs, and 55 mature HFAs. They span a wide range of magnetic local times (MLTs) from approximately 7 to 16.5 MLT. The largest ratio of solar wind to HFA core density occurred near dusk and at larger distances from the bow shock. In this study, HFAs and SHFAs were observed up to $6.3 R_{\mathrm{E}}$ and $6.1 R_{\mathrm{E}}$ (Earth radii), respectively, upstream from the model bow shock. HFA-SHFA occurrence decreases with distance upstream from the bow shock. HFAs of the highest event core ion temperatures were not seen at the flanks. The ratio of HFA ion temperature increase to HFA electron temperature increase is highest around 12 MLT and slightly duskward. For SHFAs, $\left(T_{\text {ihfa }} / T_{\text {isw }}\right) /\left(T_{\text {ehfa }} / T_{\text {esw }}\right)$ generally increased with distance from the bow shock. Both mature and young HFAs are more prevalent when there is an approximately radial interplanetary magnetic field. HFAs occur most preferentially for solar wind speeds from 550 to $600 \mathrm{~km} \mathrm{~s}^{-1}$. The correlation coefficient between the HFA increase in thermal energy density from solar wind values and the decrease in kinetic energy density from solar wind values
\end{abstract}

is 0.62. SHFAs and HFAs do not show major differences in this study.

Keywords. Interplanetary physics (planetary bow shocks; solar wind plasma) - magnetospheric physics (solar windmagnetosphere interactions)

\section{Introduction}

Hot flow anomalies (HFAs) are kinetic plasma phenomena observed near Earth's bow shock. They are characterized by a significant increase in temperature, substantial plasma flow deflection from the solar wind flow direction, and a corresponding decrease in density (Schwartz et al., 1985; Thomsen et al., 1986, 1988). HFA ions are thought to be heated from the coupling of ions reflected from the bow shock and incident solar wind ions via certain types of instabilities (Thomsen et al., 1988; Zhang et al., 2010). Investigating hot flow anomalies is important because they may contribute significantly to the solar wind-magnetosphere-ionosphere coupling. Sibeck et al. (1999) described magnetopause motion and auroral brightening related to an HFA. Eastwood et al. (2008) presented a magnetic impulse event associated with an HFA, which mapped to the dawn flank of the magnetosphere. The HFA pressure perturbation had a significant 
impact on the magnetopause location, which was measured with ground-based instruments. Fillingim et al. (2011) presented ground- and satellite-based instrument observations of auroral brightening and a traveling convection vortex associated with an HFA.

Thomas et al. (1991) showed in simulations that the interaction of a solar wind tangential discontinuity with the bow shock could result in the formation of an HFA. The observed ion temperatures inside an HFA are typically an order of magnitude above the electron temperatures $\left(T_{i} \sim 10^{7} \mathrm{~K}\right.$, $\left.T_{\mathrm{e}} \sim 10^{6} \mathrm{~K}\right)$ and the plasma flow speeds typically range from about 20 to $50 \%$ of the solar wind speed (Onsager et al., 1990).

Two distinct types of HFAs have been identified, "young" and "mature" (Thomsen et al., 1986; Lucek et al., 2004). HFA observations with both a solar wind and reflected component have been interpreted as the signatures of an HFA in an early stage of development. HFA observations with a single hot ion population have been interpreted as the signature of the later-stage evolution of a HFA (Zhang et al., 2010).

Facskó et al. (2009) found that HFAs could be identified at distances from Earth greater than $19 R_{\mathrm{E}}$. Using a model bow shock and accounting for the local solar wind, they observed HFAs greater than $4 R_{\mathrm{E}}$ upstream from the bow shock. Determining the typical locations of HFA observations will further our fundamental understanding of where conditions favor HFA existence.

HFA properties that may evolve with time include flow velocities, density, temperature, magnetic field strength, and size. Zhang et al. (2010) showed observations of a protoHFA (with decreases from solar wind levels in magnetic field strength and plasma density) and then observations of a mature HFA with the same satellite constellation $110 \mathrm{~s}$ later (hot core flanked by enhanced magnetic field strength and plasma density).

Spontaneous HFAs (SHFAs) were described by Zhang et al. (2013); Omidi et al. (2013). SHFAs exhibit the same deflected solar wind plasma velocities and plasma heating as HFAs used to classify events, but they are observed without the solar wind discontinuities that are thought to generate HFAs. Cone angle is the angle between the interplanetary magnetic field (IMF) and an axis along the Sun-Earth line. Omidi et al. (2014) showed that SHFAs in hybrid code simulations may form at all cone angles and form more frequently at higher Alfvén Mach numbers.

HFA-SHFA formation requires kinetic energy from the solar wind to be converted into thermal energy inside the event. This suggests that young HFAs-SHFAs should exhibit a smaller increase in thermal energy than mature HFAsSHFAs. Wang et al. (2013) analyzed HFAs observed by Cluster to conclude that part of the solar wind kinetic energy is converted into thermal energy inside the HFAs via heating processes.

This study compares the characteristics, such as density depletion and temperature increase, of all four categories of
HFAs: young spontaneous, young non-spontaneous, mature spontaneous, and mature non-spontaneous. We also examine the occurrence rate of HFAs and their dependence on solar wind-IMF conditions and distance from the bow shock. We expect that the HFA core density and temperatures may exhibit weak dependencies on both the distance at which the HFA is observed from the bow shock and its magnetic local time. From conclusions drawn by Facskó et al. (2009), we expect that the HFAs observed by THEMIS (Time History of Events and Macroscale Interactions during Substorms) in this study will have an occurrence rate that increases for fast solar wind speeds. Physically, at the bow shock, solar wind particles are reflected back towards the foreshock region at the solar wind speed. It is presumed that the interaction of the reflected and incoming solar wind is what forms the HFA. Higher solar wind speeds therefore mean that the reflected beam has higher energy and an increased chance that an HFA could form. We expect the occurrence rate of HFAs to decrease with increasing distance upstream from the bow shock.

We expect that the highest occurrence rates of HFAs will occur near the more radial IMF orientations because at those configurations, the particles reflected off of the bow shock are able to travel further upstream into the foreshock region and interact with the solar wind particles.

\section{Datasets and methodology}

\subsection{Event identification}

This study uses data from the THEMIS spacecraft (Angelopoulos, 2008). The five THEMIS spacecraft spent more than $10000 \mathrm{~h}$ in the interplanetary medium upstream of Earth's bow shock from 2007 to 2009 (NASA SSCWeb data). During that time, the inter-spacecraft separation varied from hundreds of kilometers to dozens of Earth radii $\left(R_{\mathrm{E}}\right)$.

Magnetic field data presented in this paper are from the fluxgate magnetometer (FGM) (Auster et al., 2008). Onboard spin resolution ( $3 \mathrm{~s})$ moment data consisting of density, velocity, ion temperature, and electron temperature came from the electrostatic analyzer (ESA) (McFadden et al., 2008).

The ion energies observed by this instrument range from a few electron volts up to $25 \mathrm{keV}$, while the electron energies range from a few electron volts up to $30 \mathrm{keV}$. For the time intervals when HFAs were observed in the solar wind by THEMIS, only a small handful of events had burst mode ESA data available. Full-mode, reduced-mode, and onboard THEMIS moment data were available. Full-mode data comes in two forms, fast mode or slow mode. Fast mode has 32 spins per data packet and slow mode has 128 spins per data packet. Both fast and slow full-mode data are too low in temporal resolution to provide multiple distribution functions for HFAs, which are typically observed by THEMIS over approximately 60 to $180 \mathrm{~s}$. Reduced-mode data have high tem- 
(a)

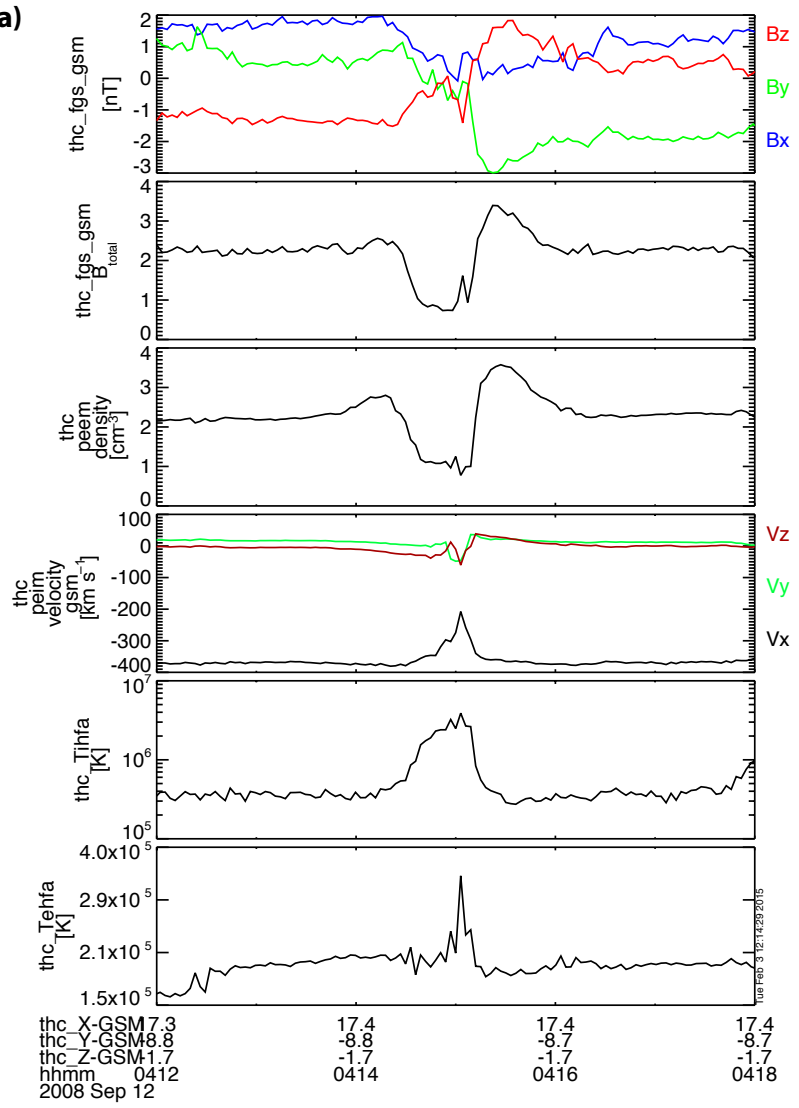

(b)

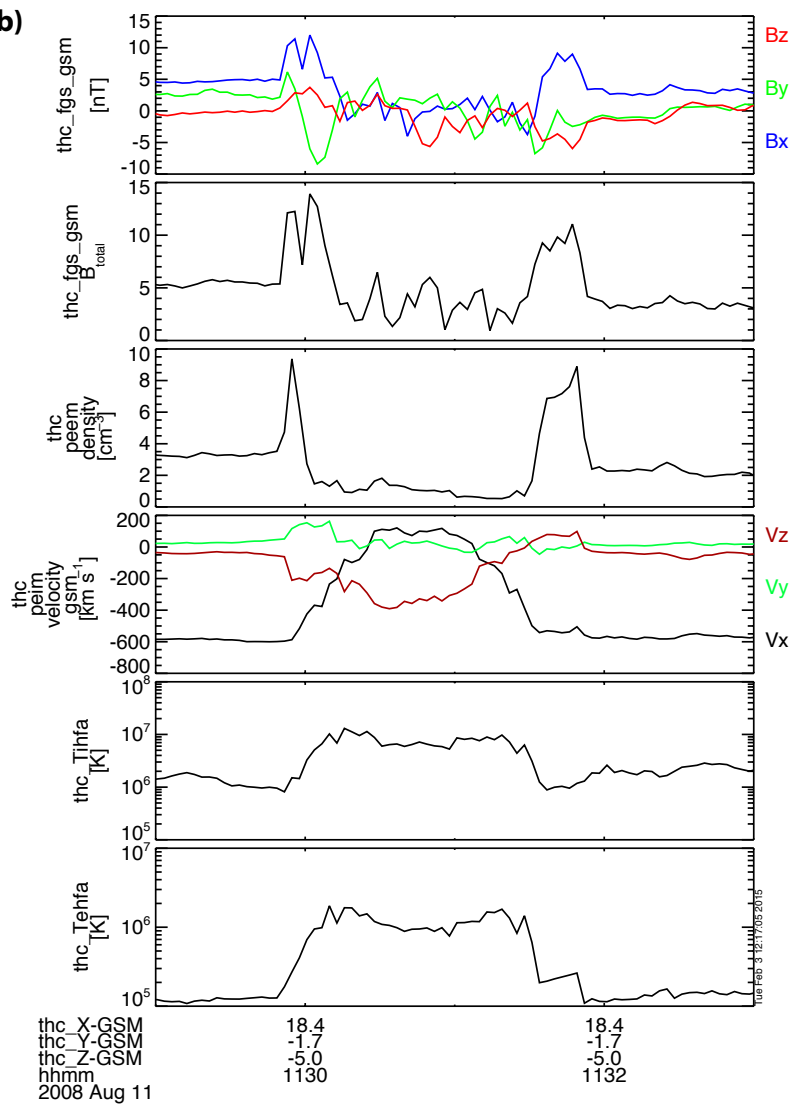

Figure 1. Plasma parameters of HFAs. Rows of the figure from top to bottom are the magnetic field components, total magnetic field magnitude, density, and particle velocity from THEMIS C onboard moment data, calculated ion temperature, and electron temperature. Parameters are shown with GSM coordinates. (a) is a young (non-spontaneous or regular) HFA and (b) is a mature spontaneous HFA.

poral $(\sim 3 \mathrm{~s})$ resolution but low angle resolution (only 6 to 50 solid angle bins of the 88 possible).

Our systematic survey of 2007-2009 THEMIS C observations identified 136 HFAs. We identified time intervals when particle flow deflections of at least $10 \%$ of the solar wind speed occurred concurrently with increased temperatures of at least $60 \%$ of the solar wind ion temperature.

HFAs were classified as young or mature by examining the magnitudes of the enhanced magnetic field strength and density enhancements bounding the core of the structures as compared to values in the solar wind. Examples of the plasma signatures of each HFA type are shown in Fig. 1a and $b$. The properties plotted are the magnetic field components, total magnetic field, density, velocity, ion temperature, and electron temperature. Mature HFAs exhibit a double peak structure in density and magnetic field strength (Fig. 1b) and have an identifiable sheath because the outer boundary of the structure is a shock (Schwartz, 1995). Young HFAs exhibit decreases in those parameters (Fig. 1a) at the core of the structure and do not have shocks at their edges. Previous work indicates that mature HFAs exhibit a single ion population, whereas young HFAs exhibit two (Zhang et al.,
2010). This criterion was not used because velocity distributions with high time $(3 \mathrm{~s})$ and angular resolution are required to resolve the two populations in young HFAs. While the full mode data have high angular resolution, the time resolution is too low to be used for this analysis.

Two other categories of HFAs are regular HFAs and SHFAs. This study used the rotation of the IMF vector to determine whether a discontinuity was observed or not. To characterize an HFA as spontaneous we first identified two solar wind intervals, one just before the event and one after. The length of the solar wind intervals were determined by starting at the outer edges of an HFA and selecting solar wind intervals. These intervals were typically $10 \mathrm{~min}$ long and did not include any non-nominal solar wind. There were times when THEMIS crossed the bow shock into the magnetosheath within $10 \mathrm{~min}$ of the hot flow anomaly boundaries. In those cases, solar wind intervals shorter than $10 \mathrm{~min}$ were selected. Then, the angle between the average solar wind magnetic field vectors before and after each HFA was calculated. If the angle was less than $30^{\circ}$, that HFA was labeled an SHFA, otherwise it was labeled a regular HFA. Figure $1 \mathrm{~b}$ shows a spontaneous HFA with a rotation angle of $29^{\circ}$, and 
(a)

THEMIS ORBIT

2007 08/10 - $200912 / 31$
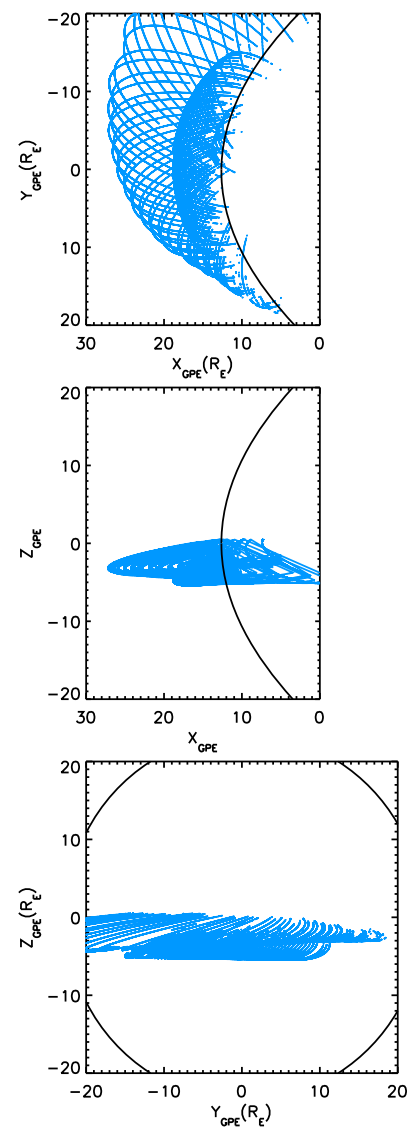

(b)

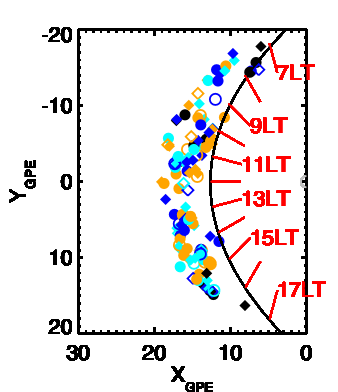

$\mathrm{Ti}[\mathrm{K}]$

Thfa $\leq 3.5 \mathrm{e}+06$

$3.5 \mathrm{e}+06<$ Thfa $\leq 6.5 \mathrm{e}+06$

$6.5 \mathrm{e}+06<$ Thfa $\leq 9.5 \mathrm{e}+06$
Thfa $>9.5 \mathrm{e}+06$

- Mature SHFA

- Moung SHFA

\& Young HFA

Figure 2. (a) The spatial location of all THEMIS C intervals in the interplanetary medium for 2007-2009 in GPE coordinates. The thick black curve is the Merka et al. (2005) bow shock for an Alfvén Mach number range of 2-20. (b) The spatial locations of HFAs-SHFAs identified in THEMIS C data, August 2007 to December 2009, are plotted in this figure in the $x y, x z$, and $y z$ planes using GPE coordinates. The thick black curves are the Merka et al. (2005) bow shock for an Alfvén Mach number range of 2-20. Four symbols represent the four HFA categories: young HFA, mature HFA, young SHFA, and mature SHFA (open diamond, filled-in diamond, open circle, and filled-in circle, respectively). The color of each HFA or SHFA corresponds to the largest temperature measured inside each HFA.

Fig. 1a shows a regular, non-spontaneous HFA with an IMF rotation angle of $89^{\circ}$.

\subsection{Bow shock model}

Solar wind flow directions vary with time, causing the bow shock location to vary with respect to the Sun-Earth line. This can make locating events with respect to the bow shock difficult. Merka and Szabo (2004) described a geocentric plasma ecliptic (GPE) coordinate system to allow comparison of separate events occurring at their corresponding bow shock crossings by accounting for effects of the solar wind flow direction. GPE is a rotation of the Geocentric Solar Ecliptic (GSE) coordinate system. In GSE, the $x$ axis points from Earth to the Sun, the $y$ axis is in the ecliptic plane pointing towards dusk (direction opposing planetary motion), and the $z$ axis is parallel to the ecliptic pole. In GPE, the $x$ axis is antiparallel to the solar wind flow, the ecliptic north is located in the $x z$ GPE plane, and $y$ is orthogonal to $x$ and $z$. GPE coordinates were used in this study to determine the distance of each event from the bow shock.

\section{Results}

Figure 2a shows the locations where THEMIS $\mathrm{C}$ made observations in the solar wind from 2007 to 2009 in the $x y$, $x z$, and $y z$ planes of the GPE coordinate system. Figure $2 b$ shows the location of 136 hot flow anomalies in the $x y, x z$, and $y z$ plane using GPE coordinates. Four symbols represent the four HFA categories: young HFA, mature HFA, young SHFA, and mature SHFA (open diamond, filled-in diamond, open circle, and filled-in circle, respectively). The color of each HFA or SHFA corresponds to the maximum ion tem- 

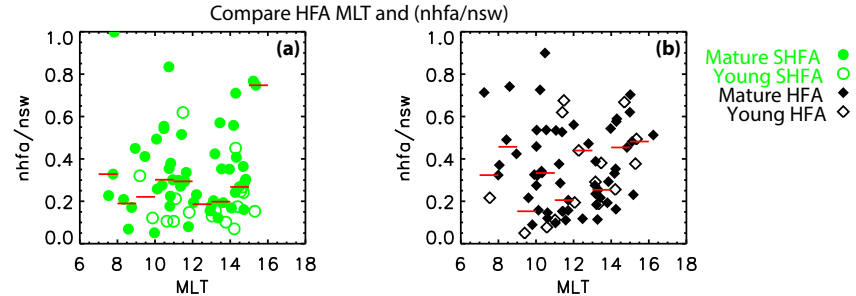

Compare HFA MLT and HFA to solar wind ion temperature ratio
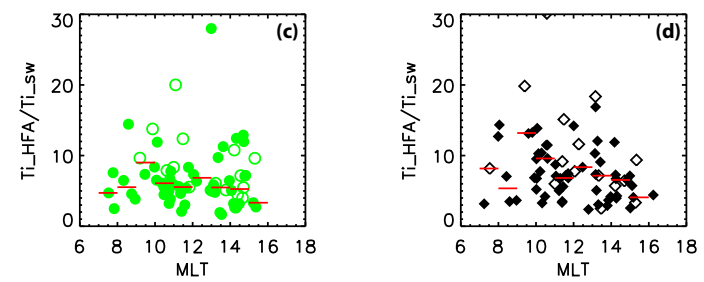

Figure 3. (a) and (b): the ratios of the minimum density of the HFA or SHFA core to the local solar wind density are plotted with respect to the events' magnetic local time (MLT). Panel (a) shows SHFAs and (b) shows HFAs. (c) and (d): ratio of ion temperatures of HFAs or SHFAs to the local solar wind ion temperature are plotted with respect to the events' MLT. Panel (c) shows SHFAs and (d) shows HFAs. Four symbols represent the four HFA categories: young HFA, mature HFA, young SHFA, and mature SHFA (open diamond, filled-in diamond, open circle, and filled-in circle, respectively). SHFAs are shown in green and HFAs are shown in black. Red lines indicate the median value of data bins along the $x$ axis.

perature observed in the core of each HFA. In this study, the observed ion temperature inside an HFA ranged from 3 to 97 times the electron temperature. The drawn bow shocks are model surfaces using coefficients from the Merka et al. (2005) bow shock model for an Alfvén Mach number range of 2-20. Merka et al. (2005) specify empirically calculated bow shocks for five individual Alfvén Mach number ranges of 2-5, 5-8, 8-13, 13-20, and 2-20. The average bow shock derived from data with the widest Mach number range, 220 , was chosen for the figures instead of a bow shock derived with Mach number ranges 2-5, 5-8, 8-13, or 13-20 so that a comparison of HFAs and SHFAs at all Mach numbers could be performed against each other. The HFAs were first divided into groups based on their local solar wind Mach number (Mach 2-5, 5-8, 8-13, and 13-20). For each Mach number range, the distance of the HFA from the corresponding model bow shock was calculated. The plotted HFA positions relative to the 2-20 Mach number model bow shock were scaled so that all HFAs were located upstream of the bow shock.

In Fig. $2 b$, the top plot with the $x y$ plane shows that HFAs were observed over the range of magnetic local times (MLTs) from approximately 7 to 16.5 MLT. The colors of Fig. 2b show the ion temperatures of the HFA cores, $T_{i}$. On the $x y$ plane of Fig. 2b, we see that the hottest ion temperatures at the core of the HFAs, shown in red, are seen within approx-

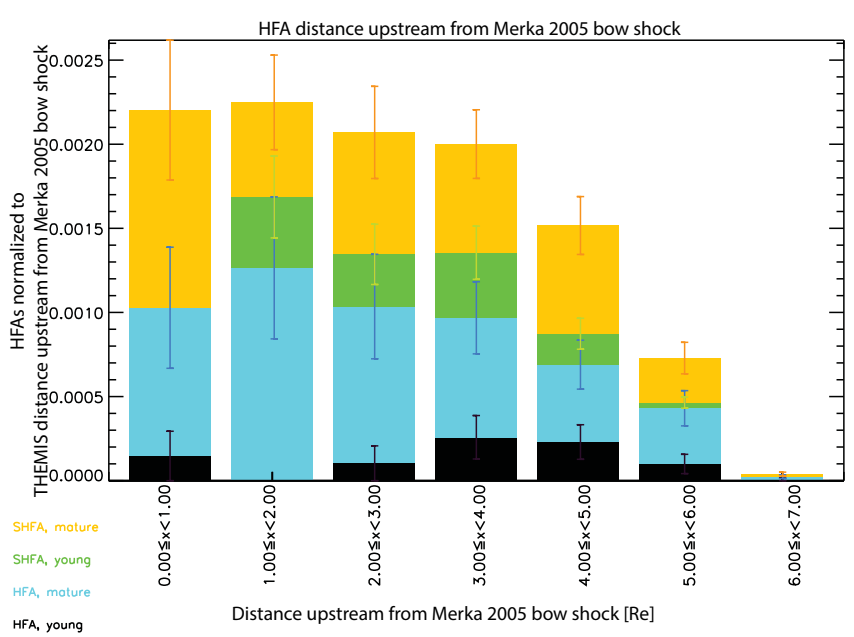

Figure 4. HFAs-SHFAs identified in THEMIS C data, August 2007 to December 2009, plotted with respect to the events' distance upstream from the Merka et al. (2005) bow shock. Events have been separated into four categories: young HFA, mature HFA, young SHFA, and mature SHFA. Each bin has been normalized to the amount of time THEMIS spent upstream of the bow shock. THEMIS made observations up to $24.5 R_{\mathrm{E}}$ upstream of the bow shock.

imately $8-15$ MLT and not seen at the far dawn and dusk flanks outside the $8-15$ MLT range.

Figure 3 a shows $n_{\mathrm{HFA}} / n_{\mathrm{sw}}$ with respect to MLT for young and mature SHFAs, and Fig. $3 \mathrm{~b}$ shows $n_{\mathrm{HFA}} / n_{\mathrm{sw}}$ with respect to MLT for young and mature regular HFAs. $n_{\mathrm{HFA}}$ is the minimum density value observed for each HFA event. The red horizontal lines represent the median value of the HFAs' $n_{\mathrm{HFA}} / n_{\mathrm{sw}}$ in the MLT range spanned by each red line. $n_{\mathrm{HFA}} / n_{\mathrm{Sw}}$ does not seem to show a dependance on MLT.

Figure $3 c$ shows the ratio of the hottest measured ion temperature of each HFA to the average local solar wind ion temperature plotted with respect to MLT for young and mature SHFAs, while Fig. 3d shows the ratio of HFA to local solar wind ion temperature for young and mature regular HFAs plotted against MLT. The red horizontal lines represent the median value of the ratio of HFA to local solar wind ion temperature in the MLT range spanned by each red line. HFAs with high event core ion temperatures or large ratio values were not seen at the dawn and dusk flanks. This may suggest that HFAs cool as they convect with the solar wind towards the flanks. It may also suggest that HFAs form with lower core temperatures away from the subsolar region.

Figure 4 shows the HFAs of the dataset binned by distance upstream from the Merka et al. (2005) model bow shock, where the distance was measured along the normal to the bow shock.

These distances are calculated using the model bow shock coefficients that correspond to the Alfvén Mach number ranges $2-5,5-8,8-13$, or $13-20$. The bins are normalized by the amount of time that THEMIS $\mathrm{C}$ spent in the respective 
Compare HFA distance from bow shock and nhfa/nsw

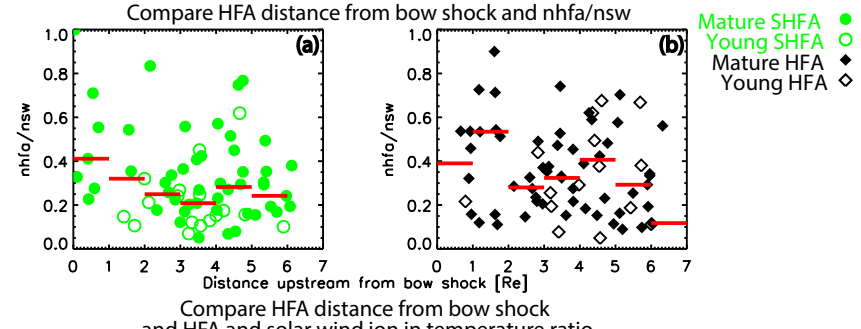

and HFA and solar wind ion in temperature ratio

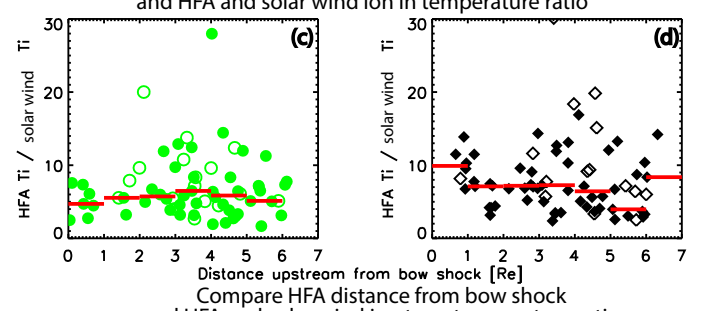

and HFA and solar wind ion to e- temperature ratio

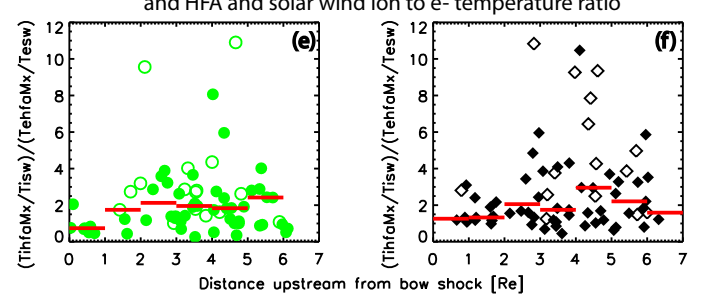

Figure 5. (a) and (b): the ratios of the minimum density of the HFA or SHFA core to the local solar wind density are plotted with respect to the events' distance upstream from the Merka et al. (2005) bow shock. Panel (a) shows SHFAs and (b) shows HFAs. (c) and (d): the ratios of the maximum event ion temperature to the events' average solar wind ion temperature, $\left(T_{\mathrm{ihfa}} / T_{\mathrm{isw}}\right)$, are plotted in this figure with respect to the events' distance upstream from the Merka et al. (2005) bow shock. Panel (c) shows SHFAs and (d) shows HFAs. (e) and (f): the ratio of the events' maximum event ion temperature to the events' average solar wind ion temperature relative to the ratio of the events' maximum event electron temperature to the events' average solar wind electron temperature, $\left(T_{\text {ihfa }} / T_{\text {isw }}\right) /\left(T_{\text {ehfa }} / T_{\text {esw }}\right)$, are plotted in this figure with respect to the events' distance upstream from the Merka et al. (2005) bow shock. Panel (e) shows SHFAs and (f) shows HFAs. Four symbols represent the four HFA categories: young HFA, mature HFA, young SHFA, and mature SHFA (open diamond, filled-in diamond, open circle, and filled-in circle, respectively). SHFAs are shown in green and HFAs are shown in black. Red lines indicate the median value of data bins along the $x$ axis.

bin's range from 2007 to 2009. HFAs and SHFAs were observed up to $6.3 R_{\mathrm{E}}$ and $6.1 R_{\mathrm{E}}$ upstream from the bow shock, respectively. It is possible that proto-HFAs were present even further upstream from the bow shock but they did not exhibit large enough plasma parameter deflections for identification as an HFA or SHFA. HFAs and SHFAs both show similar trends where the occurrence decreases with increasing distance upstream from the bow shock.

Figure $5 \mathrm{a}$ and $\mathrm{b}$ show $n_{\mathrm{HFA}} / n_{\mathrm{sw}}$ with respect to each event's distance from the Merka et al. (2005) model bow shock for young and mature SHFAs (a) and young and ma- ture regular HFAs (b). The red horizontal lines represent the median value of $n_{\mathrm{HFA}} / n_{\mathrm{sw}}$ in the bow shock distance range spanned by each red line. The median values of $n_{\mathrm{HFA}} / n_{\mathrm{sw}}$ generally decrease with distance from the bow shock for SHFAs from $0 R_{\mathrm{E}}$ to $4 R_{\mathrm{E}}$ upstream from the bow shock but over all distances does not show a clear trend.

For the HFA dataset in this study, when an HFA forms near the bow shock, its core density has a median ratio of approximately 0.4 to the nearby solar wind's density. As an HFA's distance upstream from the bow shock increases, the ratio of core density to solar wind density decreases.

Figure $5 \mathrm{c}$ and $\mathrm{d}$ plot the ratio of the hottest ion temperature observed in each HFA to the average solar wind temperature, $T_{\text {ihfa }} / T_{\text {isw }}$, against the HFA's distance from the Merka et al. (2005) bow shock. Young and mature SHFAs are shown in Fig. 5c and young and mature regular HFAs are shown in Fig. 5d. The red horizontal lines represent the median value of $T_{\text {ihfa }} / T_{\text {isw }}$ in the bow shock distance range spanned by each red line. The absolute hottest ion heating for both SHFAs and HFAs is located at around $4 R_{\mathrm{E}}$.

Examining the red median lines, the conclusion is that there was no clear relationship between the ratio of HFA to solar wind temperature and distance at which a HFA was observed upstream from the bow shock.

Figure 5e and f examine how much the ion temperature increased inside each HFA compared to how much the electron temperature increased inside the HFA $\left(\left(T_{\text {ihfa }} / T_{\text {isw }}\right) /\left(T_{\text {ehfa }} / T_{\text {esw }}\right)\right)$ and plots that against distance from the Merka et al. (2005) bow shock. Young and mature SHFAs are shown in Fig. 5e and young and mature regular HFAs are shown in Fig. 5f. The red horizontal lines represent the median value of $\left(\left(T_{\text {ihfa }} / T_{\text {isw }}\right) /\left(T_{\text {ehfa }} / T_{\text {esw }}\right)\right)$ in the bow shock distance range spanned by each red line. Significant differences between the ion and electron heating ratios was not expected. The ion heating relative to the electron heating was highest between 2 and $6 R_{\mathrm{E}}$ for both SHFAs and HFAs, but this was only a slight increase in heating from HFAs in the other distance bins.

In Fig. 5e, examining the red median lines for the SHFAs reveals that $\left(\left(T_{\mathrm{ihfa}} / T_{\text {isw }}\right) /\left(T_{\text {ehfa }} / T_{\text {esw }}\right)\right)$ generally increased with distance from the bow shock. For HFAs in Fig. 5f, $\left(\left(T_{\text {ihfa }} / T_{\text {isw }}\right) /\left(T_{\text {ehfa }} / T_{\text {esw }}\right)\right)$ generally increased with distance from the bow shock until about $5 R_{\mathrm{E}}$ and then decreased. For SHFAs, the factor that ions were heated by, $\left(T_{\mathrm{ihfa}} / T_{\mathrm{isw}}\right)$, approximately equaled the factor that electrons were heated by, $\left(T_{\text {ehfa }} / T_{\text {esw }}\right)$, for $0-1 R_{\text {E }}$ so that $\left(\left(T_{\text {ihfa }} / T_{\text {isw }}\right) /\left(T_{\text {ehfa }} / T_{\text {esw }}\right)\right)$ was about equal to 1 . As HFAs were observed further from the bow shock, the factor that ions were heated by was greater than the factor that electrons were heated by.

For both SHFAs and HFAs, from 0 to $2 R_{\mathrm{E}}$, the median value of the ratio $\left(\left(T_{\text {ihfa }} / T_{\text {isw }}\right) /\left(T_{\text {ehfa }} / T_{\text {esw }}\right)\right)$ is slightly lower than for 2-7 $R_{\mathrm{E}}$. From approximately 0 to $1 R_{\mathrm{E}}$ upstream of the bow shock, the amount of ion heating, ( $\left.T_{\text {ihfa }} / T_{\text {isw }}\right)$, is the same as the electron heating, $\left(T_{\text {ehfa }} / T_{\text {esw }}\right)$. From 1 to $7 R_{\mathrm{E}}$, the electron heating ratio, $\left(T_{\mathrm{ehfa}} / T_{\mathrm{esw}}\right)$, 


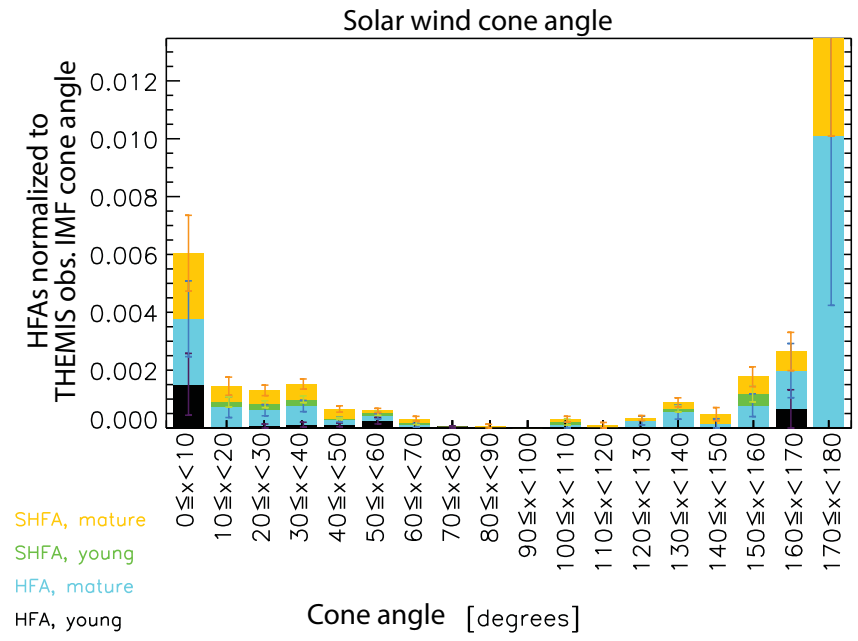

Figure 6. Analysis of HFAs and SHFAs with respect to IMF cone angle. The 136 HFAs were separated into four categories (young HFA, mature HFA, young SHFA, and mature SHFA) and were binned with respect to the average IMF cone angle in the local solar wind for each event. Each bin was normalized with respect to the amount of time that THEMIS C saw solar wind of that angle from 2007 to 2009. Color-coded error bars for each HFA category are plotted.

was lower than the ion heating ratio, $\left(T_{\mathrm{ihfa}} / T_{\mathrm{isw}}\right)$, so that $\left(\left(T_{\text {ihfa }} / T_{\text {isw }}\right) /\left(T_{\text {ehfa }} / T_{\text {esw }}\right)\right)$ was greater than 1 .

Figure 6 examines the relationship between HFAs-SHFAs and the IMF cone angle (the angle between the SunEarth line and $\left.B_{\mathrm{IMF}}\right)$. Cone angle $\theta$ is defined as $\theta=$ $\cos ^{-1}\left(\frac{b_{x}}{\sqrt{b_{x}^{2}+b_{y}^{2}+b_{z}^{2}}}\right)$. THEMIS C observed solar wind intervals both before and after each HFA so that two different cone angles could be calculated for each event, one averaging the solar wind before observing the HFA and one after. To create this figure, both angles were calculated and the most radial cone angle was chosen as the sole solar wind cone angle value for the particular HFA (the angle closest to 0 or $180^{\circ}$ was chosen). The highest normalized occurrence rates of HFAs and SHFAs are for cone angles between 0 and $40^{\circ}$ and from 160 to $180^{\circ}$. This figure shows that HFAs form more preferentially for more radial IMF configurations and 0 and $180^{\circ}$ correspond to radial IMF conditions.

Figure 7 shows HFA occurrence normalized to solar wind speeds. First, the number of HFAs were binned according to the local solar wind speed near HFA observations. The local solar wind speed was calculated using the solar wind intervals for each event, as defined in Sect. 2.1. Then the number of events in each bin was normalized by the total amount of time that THEMIS C observed solar wind of that speed during the years of 2007-2009. The peak normalized occurrence rate of HFAs and SHFAs occurred for solar wind speed of 550-600 $\mathrm{km} \mathrm{s}^{-1}$. Facskó et al. (2009) noted in their study that HFA formation is more prevalent for faster solar
Table 1. Percentage of HFAs with radial IMF in different solar wind speed ranges.

\begin{tabular}{lr}
\hline Solar wind speed & $\begin{array}{r}\text { Percentage of HFAs } \\
\text { with radial IMF }\end{array}$ \\
\hline$x<300 \mathrm{~km} \mathrm{~s}^{-1}$ & $0 \%$ \\
$300 \mathrm{~km} \mathrm{~s}^{-1} \leq x<350 \mathrm{~km} \mathrm{~s}^{-1}$ & $14 \%$ \\
$350 \mathrm{~km} \mathrm{~s}^{-1} \leq x<400 \mathrm{~km} \mathrm{~s}^{-1}$ & $12 \%$ \\
$400 \mathrm{~km} \mathrm{~s}^{-1} \leq x<450 \mathrm{~km} \mathrm{~s}^{-1}$ & $10 \%$ \\
$450 \mathrm{~km} \mathrm{~s}^{-1} \leq x<500 \mathrm{~km} \mathrm{~s}^{-1}$ & $18 \%$ \\
$500 \mathrm{~km} \mathrm{~s}^{-1} \leq x<550 \mathrm{~km} \mathrm{~s}^{-1}$ & $5 \%$ \\
$550 \mathrm{~km} \mathrm{~s}^{-1} \leq x<600 \mathrm{~km} \mathrm{~s}^{-1}$ & $22 \%$ \\
$x \geq 600 \mathrm{~km} \mathrm{~s}^{-1}$ & $40 \%$ \\
\hline
\end{tabular}

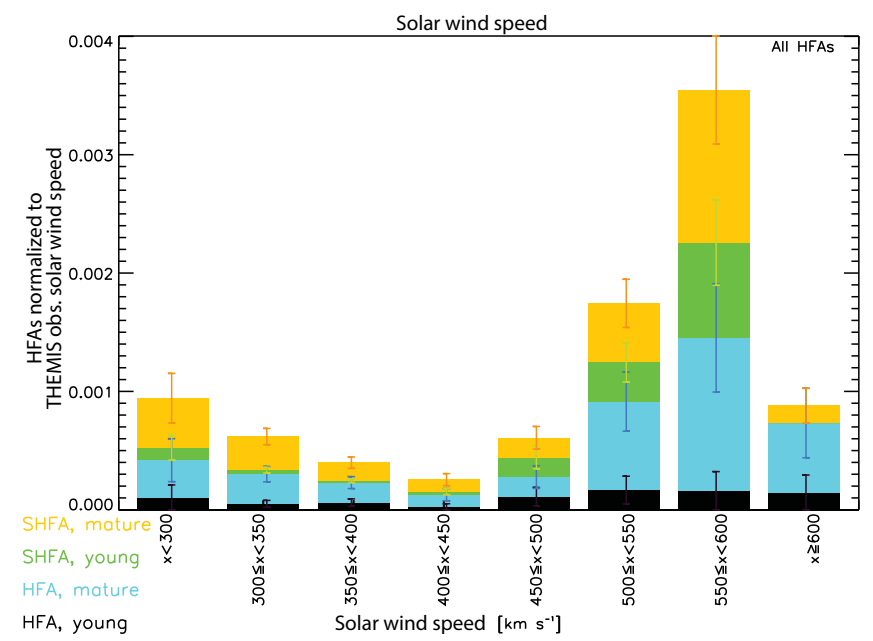

Figure 7. The number of HFAs in each solar wind speed bin is normalized by the amount of time there is solar wind of that speed. Four colors represent the four HFA categories: young HFA, mature HFA, young SHFA, and mature SHFA. Error bars for each of the four HFA categories are plotted.

wind speeds, a point which this dataset agrees with for 400 $600 \mathrm{~km} \mathrm{~s}^{-1}$.

In Fig. 7, the HFA occurrence rate decreases with increasing solar wind speeds from 0 to $400 \mathrm{~km} \mathrm{~s}^{-1}$. To explain this observation, we performed an analysis to separate the IMF orientation effect on occurrence rates from the effects of solar wind speed. From Fig. 6, we observed that HFAs occur preferentially when the IMF is radial. Radial IMF was defined as times when the IMF had a cone angle between 0 and $15^{\circ}$ or 165 and $180^{\circ}$. For each solar wind speed bin in Fig. 7, we calculated the percentage of HFAs in that bin that had radial IMF. Results are listed in Table 1. In Table 1, $x$ is defined as each event's solar wind speed. The bins of $x<300 \mathrm{~km} \mathrm{~s}^{-1}$ and $x \geq 600 \mathrm{~km} \mathrm{~s}^{-1}$ have too few events in them to be statistically significant and were ignored. We observed decreasing occurrence rates from 300 to $450 \mathrm{~km} \mathrm{~s}^{-1}$ in Fig. 7, which corresponds to rows $300-350,350-400$, and $400-450 \mathrm{~km} \mathrm{~s}^{-1}$ in 


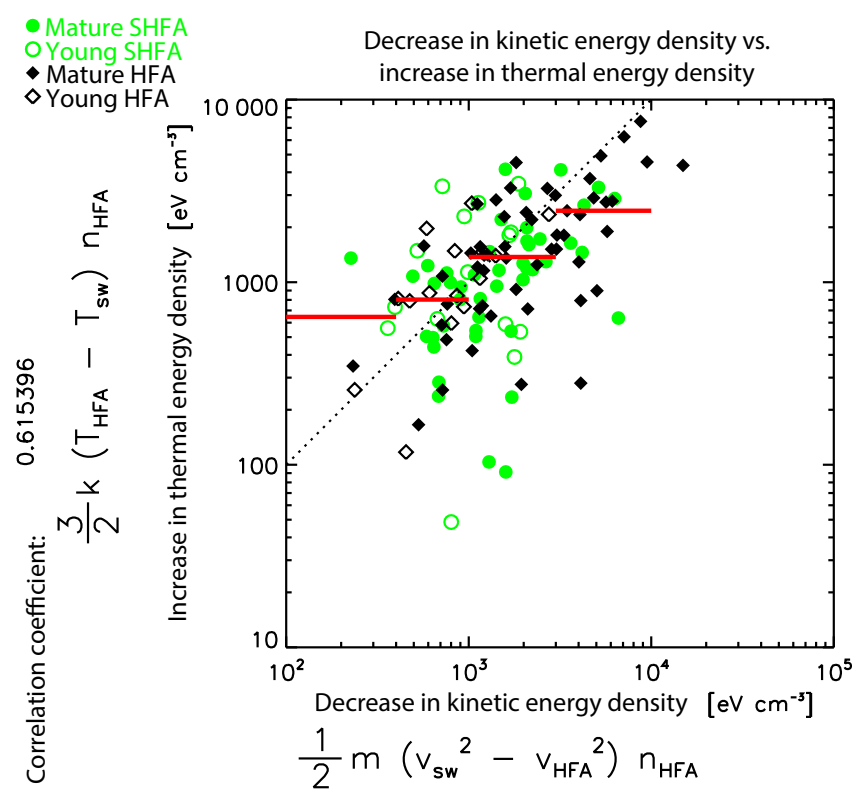

Figure 8. This figure plots the thermal energy increase of each HFA or SHFA compared to its kinetic energy decrease (as compared to the solar wind). The HFAs-SHFAs are identified from THEMIS C data from 2008. The green and black symbols refer to SHFAs and HFAs, respectively. Open and filled symbols refer to young and mature events, respectively. The dotted black line in the plot is the oneto-one diagonal. Red horizontal lines mark the median value of data bins along the $x$ axis.

Table 1. The percentage of HFAs with radial IMF in these bins decreases as solar wind speed increases $(14,12,10 \%)$. The decrease in the HFA occurrence rate with increasing solar wind speed in the range of $300-450 \mathrm{~km} \mathrm{~s}^{-} 1$ comes from a decrease in the number of radial IMF HFAs at those solar wind speeds. For speeds between 400 and $600 \mathrm{~km} \mathrm{~s}^{-1}$, the percentages in the table do not follow their respective bins in Fig. 7. We put forth that HFA occurrence rate is not as highly related to IMF orientation at fast solar wind speeds because fast solar wind creates other favorable HFA formation conditions. The speed of backstreaming particles is faster for faster solar wind speeds. The thermal energy in HFAs mainly comes from the coupling between the reflected beam and the original solar wind beam. This causes the relative speed between the two beams to be crucial for HFA formation.

Figure 8 plots the thermal energy density increase within each HFA and SHFA versus the corresponding kinetic energy density decrease (as compared to the solar wind in both cases). The correlation coefficient is 0.62 . The HFA kinetic and thermal energy are both calculated at the time when the flow deflection inside the HFA is the strongest. The green and black symbols refer to SHFAs and HFAs, respectively. Open and filled symbols refer to young and mature events, respectively. The black dotted line in the plot is the one-toone diagonal. Red lines indicate the median value of data bins along the $x$ axis. Some young HFAs and SHFAs have a smaller increase in thermal energy than mature HFAs and SHFAs, but this is not seen for all cases in this figure. This leads to the hypothesis that some of the mature HFAs and SHFAs in our study are events that have existed for a while and have therefore cooled through some mechanism such as adiabatic expansion. HFAs expand when the internal pressure is high. When they cool down, the internal pressure decreases. SHFAs and HFAs do not show differences in this analysis of an event's kinetic and thermal energies relative to the local medium.

\section{Conclusions}

In this study, 136 HFAs (104 mature, 32 young; 66 spontaneous, 70 regular) were identified from THEMIS C data from August 2007 to December 2009. The dataset has 17 young SHFAs, 49 mature SHFAs, 15 young HFAs, and 55 mature HFAs. They span a wide range of MLTs. In this study, HFAs and SHFAs were observed up to $6.3 R_{\mathrm{E}}$ and $6.1 R_{\mathrm{E}}$ upstream from the bow shock, respectively. Particles reflected from the bow shock have a limited distance that they can travel sunward because of the impinging background flow and that limits how far HFAs and SHFAs can be observed upstream from the bow shock. HFA and SHFA occurrence decreases with distance upstream from the bow shock, which has not been reported in previous literature. HFAs with the highest event core ion temperatures were not seen at the flanks. The ratio of HFA ion temperature increase to HFA electron temperature increase is highest around 12 MLT and slightly duskward. Both mature and young HFAs are slightly more prevalent when there is an approximately radial interplanetary magnetic field (Fig. 6). HFAs occur more often for large cone angles. HFAs occur most preferentially for solar wind speeds from 550 to $600 \mathrm{~km} \mathrm{~s}^{-1} . n_{\mathrm{HFA}} / n_{\mathrm{sw}}$ shows no clear trend with distance from the bow shock. By examining the thermal and kinetic energy of the events, it is hypothesized that some of the mature HFAs and SHFAs in our study are most likely events that have existed for a while before observation by THEMIS and have therefore cooled through some mechanism such as adiabatic expansion. SHFAs and HFAs do not show major differences in this study.

Data availability. This study used data from the THEMIS satellite C in the years 2007, 2008, and 2009. THEMIS data and the latest calibration files are publicly available at http://themis.ssl.berkeley. edu/ or via the SPEDAS software (Auster et al., 2008; McFadden et al., 2008; Angelopoulos, 2008).

Competing interests. The authors declare that they have no conflict of interest. 
Acknowledgements. C. Chu wishes to thank the NASA Harriet G. Jenkins Graduate Fellowship Program for their support with grant NNX13AR91H. This work was partially supported by NSF grants AGS-1007449, AGS-1303689, AGU-1303596, and AGS1352669 .

We acknowledge NASA contract NAS5-02099 and V. Angelopoulos for use of data from the THEMIS Mission. Specifically, we thank C. W. Carlson and J. P. McFadden for use of ESA data and K. H. Glassmeier, U. Auster, and W. Baumjohann for the use of FGM data provided under the lead of the Technical University of Braunschweig and with financial support through the German Ministry for Economy and Technology and the German Center for Aviation and Space (DLR) under contract 50 OC 0302.

The topical editor, E. Roussos, thanks L. Zhao and the one anonymous referee for help in evaluating this paper.

\section{References}

Angelopoulos, V.: The THEMIS Mission, Space Sci. Rev., 141, 5-34, doi:10.1007/s11214-008-9336-1, 2008 (data available at: http://themis.ssl.berkeley.edu/).

Auster, H. U., Glassmeier, K. H., Magnes, W., Aydogar, O., Baumjohann, W., Constantinescu, D., Fischer, D., Fornacon, K. H., Georgescu, E., Harvey, P., Hillenmaier, O., Kroth, R., Ludlam, M., Narita, Y., Nakamura, R., Okrafka, K., Plaschke, F., Richter, I., Schwarzl, H., Stoll, B., Valavanoglou, A., and Wiedemann, M.: The THEMIS Fluxgate Magnetometer, Space Sci. Rev., 141, 235-264, doi:10.1007/s11214-008-9365-9, 2008 (data available at: http://themis.ssl.berkeley.edu/).

Eastwood, J. P., Sibeck, D. G., Angelopoulos, V., Phan, T. D., Bale, S. D., McFadden, J. P., Cully, C. M., Mende, S. B., Larson, D., Frey, S., Carlson, C. W., Glassmeier, K., Auster, H. U., Roux, A., and Le Contel, O.: THEMIS observations of a hot flow anomaly: Solar wind, magnetosheath, and ground-based measurements, Geophys. Res. Lett., 35, L17S03, doi:10.1029/2008GL033475, 2008

Facskó, G., Trotignon, J. G., Dandouras, I., Lucek, E. A., and Daly, P. W.: Study of hot flow anomalies using Cluster multi-spacecraft measurements, Adv. Space Res., 45, 541-552, doi:10.1016/j.asr.2009.08.011, 2009.

Fillingim, M. O., Eastwood, J. P., Parks, G. K., Angelopoulos, V., Mann, I. R., Mende, S. B., and Weatherwax, A. T.: Polar UVI and THEMIS GMAG observations of the ionospheric response to a hot flow anomaly, J. Atmos. Sol.-Terr. Phy., 73, 137-145, doi:10.1016/j.jastp.2010.03.001, 2011.

Lucek, E. A., Horbury, T. S., Balogh, A., Dandouras, I., and Rème, H.: Cluster observations of hot flow anomalies, J. Geophys. Res., 109, A06207, doi:10.1029/2003JA010016, 2004.

McFadden, J. P., Carlson, C. W., Larson, D., Ludlam, M., Abiad, R., Elliott, B., Turin, P., Marckwordt, M., and Angelopoulos, V.: The THEMIS ESA Plasma Instrument and In-flight Calibration, Space Sci. Rev., 141, 277-302, doi:10.1007/s11214-008-9440-2, 2008 (data available at: http://themis.ssl.berkeley.edu/).

Merka, J. and Szabo, A.: Bow shock's geometry at the magnetospheric flanks, J. Geophys. Res.-Space, 109, A12224, doi:10.1029/2004JA010567, 2004.
Merka, J., Szabo, A., Slavin, J. A., and Peredo, M.: Threedimensional position and shape of the bow shock and their variation with upstream Mach numbers and interplanetary magnetic field orientation, J. Geophys. Res.-Space, 110, 4202, doi:10.1029/2004JA010944, 2005.

Omidi, N., Zhang, H., Sibeck, D., and Turner, D.: Spontaneous hot flow anomalies at quasi-parallel shocks: 2. Hybrid simulations, J. Geophys. Res.-Space, 118, 173-180, doi:10.1029/2012JA018099, 2013.

Omidi, N., Zhang, H., Chu, C., Sibeck, D., and Turner, D.: Parametric dependencies of spontaneous hot flow anomalies, J. Geophys. Res.-Space, 119, 9823-9833, doi:10.1002/2014JA020382, 2014.

Onsager, T. G., Thomsen, M. F., Gosling, J. T., and Bame, S. J.: Observational test of a hot flow anomaly formation mechanism, J. Geophys. Res., 95, 11967-11974, 1990.

Schwartz, S. J.: Hot flow anomalies near the Earth's bow shock, Adv. Space Res., 15, 107-116, doi:10.1016/02731177(95)00025-A, 1995.

Schwartz, S. J., Chaloner, C. P., Hall, D. S., Christiansen, P. J., and Johnstones, A. D.: An active current sheet in the solar wind, Nature, 318, 269-271, doi:10.1038/318269a0, 1985.

Sibeck, D. G., Borodkova, N. L., Schwartz, S. J., Owen, C. J., Kessel, R., Kokubun, S., Lepping, R. P., Lin, R., Liou, K., Lühr, H., McEntire, R. W., Meng, C.-I., Mukai, T., Nemecek, Z., Parks, G., Phan, T. D., Romanov, S. A., Safrankova, J., Sauvaud, J.-A., Singer, H. J., Solovyev, S. I., Szabo, A., Takahashi, K., Williams, D. J., Yumoto, K., and Zastenker, G. N.: Comprehensive study of the magnetospheric response to a hot flow anomaly, J. Geophys. Res., 104, 4577-4594, doi:10.1029/1998JA900021, 1999.

Thomas, V. A., Winske, D., Thomsen, M. F., and Onsager, T. G.: Hybrid simulation of the formation of a hot flow anomaly, J. Geophys. Res., 96, 11625-11632, doi:10.1029/91JA01092, 1991.

Thomsen, M. F., Gosling, J. T., Fuselier, S. A., Bame, S. J., and Russell, C. T.: Hot, diamagnetic cavities upstream from the earth's bow shock, J. Geophys. Res., 91, 2961-2973, doi:10.1029/JA091iA03p02961, 1986.

Thomsen, M. F., Gosling, J. T., Bame, S. J., Quest, K. B., and Russell, C. T.: On the origin of hot diamagnetic cavities near the earth's bow shock, J. Geophys. Res., 93, 11311-11325, doi:10.1029/JA093iA10p11311, 1988.

Wang, S., Zong, Q., and Zhang, H.: Hot flow anomaly formation and evolution: Cluster observations, J. Geophys. Res.-Space, 118, 4360-4380, doi:10.1002/jgra.50424, 2013.

Zhang, H., Sibeck, D. G., Zong, Q.-G., Gary, S. P., McFadden, J. P., Larson, D., Glassmeier, K.-H., and Angelopoulos, V.: Time History of Events and Macroscale Interactions during Substorms observations of a series of hot flow anomaly events, J. Geophys. Res.-Space, 115, A12235, doi:10.1029/2009JA015180, 2010.

Zhang, H., Sibeck, D. G., Zong, Q.-G., Omidi, N., Turner, D., and Clausen, L. B. N.: Spontaneous hot flow anomalies at quasiparallel shocks: 1. Observations, J. Geophys. Res.-Space, 118, 3357-3363, doi:10.1002/jgra.50376, 2013. 